\title{
Dispersion in a Gas Filled Hollow Core Photonic Crystal Fiber
}

\author{
Hanan J. Taher * \\ Received 23, April, 2013 \\ Accepted 23, September, 2013
}

\begin{abstract}
:
Hollow core photonic bandgap fibers provide a new geometry for the realization and enhancement of many nonlinear optical effects. Such fibers offer novel guidance and dispersion properties that provide an advantage over conventional fibers for various applications. Dispersion, which expresses the variation with wavelength of the guided-mode group velocity, is one of the most important properties of optical fibers. Photonic crystal fibers (PCFs) offer much larger flexibility than conventional fibers with respect to tailoring of the dispersion curve. This is partly due to the large refractive-index contrast available in the silica/air microstructures, and partly due to the possibility of making complex refractive-index structure over the fiber cross section. In this paper the fundamental physical mechanism has been discussed determining the dispersion properties of PCFs, and the dispersion in a gas filled hollow core photonic crystal fiber has been calculated.

We calculate the dispersion of air filled hollow core photonic crystal fiber, also calculate the dispersion of $\mathrm{N}_{2}$ gas filled hollow core photonic crystal fiber and finally we calculate the dispersion of $\mathrm{He}$ gas filled hollow core photonic crystal fiber.
\end{abstract}

Key words: Photonic crystal fiber; Dispersion properties of photonic crystal fibers

\section{Introduction:}

The requirements of large bandwidth and miniaturization of circuits lead to the development of periodic dielectric structures that exhibit band gaps in the frequency spectrum. These structures are commonly known as photonic crystals or photonic bandgap materials in the literature. Photonic crystals are artificial dielectric structures with the property of rejecting a frequency band of electromagnetic waves while transmitting frequencies outside the bandgap. Such crystals are the optical analog of semiconductors, because their periodic dielectric structure produces a photonic bandgap [1]. The presence of photonic bandgap opens the possibility for a variety of applications, ranging from optical communication and integrated photonic to quantum information science [2].

\section{Theory and Physical Mechanisms Determining Dispersion Behavior}

In telecommunication systems, information is transmitted as binary data, taking the form of light pulses in optical fibers. In the field of optical waveguides, dispersion is a generic term referring to all phenomena causing these pulses to spread while propagating and they eventually overlap and light pulses could not be distinguished by the receiver [3] .There are essentially three causes of dispersion .

*Institute of laser for postgraduate studies. 


\subsection{Chromatic (Intramodal) Dispersion}

Chromatic dispersion is an important phenomenon in the propagation of short pulses in optical fibers. Temporally short pulses have a large spectral bandwidth. The different spectral components of the pulse travel through the medium at slightly different group velocities because of chromatic dispersion, which can result in a temporal broadening of the light pulses with no effect on their spectral compositions. This phenomenon is referred to as group velocity dispersion (GVD), [4].

\subsubsection{Material Dispersion}

GVD is an important effect because when a short pulse propagates through an optical fiber its pulse width gets broaden. The effects arise from the variation of the refractive index of the material as a function of wavelength. This causes a wavelength dependence of the group velocity of any given mode, that is, pulse spreading occurs even when different wavelengths follow the same path [3].This phenomena can be understood by expanding the mode-propagation constant $\beta$ in a Taylor series about the frequency $\omega_{0}$ at which the pulse spectrum is centered, $[4,5]$.

$$
\begin{aligned}
& \beta(\omega)=n_{e f f}(\omega) \frac{\omega}{c}=\beta_{o}+\left(\omega-\omega_{o}\right) \beta_{1}+ \\
& \frac{1}{2}\left(\omega-\omega_{o}\right)^{2} \beta_{2}+\frac{1}{6}\left(\omega-\omega_{o}\right)^{3} \beta_{3}+\ldots
\end{aligned}
$$

Where

$$
\begin{aligned}
& \beta_{m}=\left(\frac{d^{m} \beta}{d \omega^{m}}\right)_{\omega=\omega_{o}} \quad(m=1,2, \ldots) \ldots \\
& \beta_{o}=n_{e f f}\left(\omega_{o}\right) \frac{\omega_{o}}{c} \\
& \beta_{I}=\frac{1}{c}\left(n e f f+\omega \frac{d n_{e f f}}{d \omega}\right)=\frac{1}{v_{g}}=\frac{n_{g}}{c} \ldots
\end{aligned}
$$

$$
\beta_{2}=\frac{1}{c}\left(2 \frac{d n_{e f f}}{d \omega}+\omega \frac{d^{2} n_{e f f}}{d \omega^{2}}\right)=\frac{d}{d \omega}\left(\frac{1}{v_{g}}\right) \ldots
$$

where $\boldsymbol{\beta}_{\mathrm{o}}$ is the mode-propagation constant of frequency $\omega_{0}, v_{\mathrm{g}}$ is the group velocity, and $\boldsymbol{n}_{g}$ is the group index. The group velocity is the speed of the envelope of an optical pulse propagating in a fiber. The coefficient $\boldsymbol{\beta}_{2}$ determines the changes in the group velocity of an optical pulse as a function of optical frequency. This phenomenon is known as GVD and is responsible for pulse broadening. Thus, $\boldsymbol{\beta}_{2}$ are called the GVD parameter. In general, we must retain terms up to the second-order dispersion $\boldsymbol{\beta}_{2}$ to describe pulse propagation in dispersive media, and for ultrashort pulses or those with a wide frequency spectrum it may sometimes be necessary to also include higher order terms.

The dispersion parameter $\mathbf{D}$ is commonly used in place of $\boldsymbol{\beta}_{2}$ to describe the total dispersion of a single mode fiber. It is related to $\boldsymbol{\beta}_{\mathbf{2}}$ by the relation

$$
D \cong \frac{d \beta_{1}}{d \lambda}=-\frac{2 \pi}{\lambda^{2}} \beta_{2}
$$

Where $\beta_{0}, \beta_{1}$ and $\beta_{2}$ are constants. The coefficient $\beta_{0}$ is the mode-propagation constant of frequency $\omega_{0}$ and the coefficient $\beta_{2}$ determines the changes in the group velocity of an optical pulse as a function of optical frequency. Thus, $\boldsymbol{\beta}_{2}$ are called the GVD parameter. And D in equation (6) is expressed in unit of (ps.nm/km).

GVD is an important effect because when a short pulse propagates through an optical fiber its pulse width gets broaden. The effects arise from the variation of the refractive index of the material as a function of wavelength. Since GVD mainly comes from the 
combined effects of material and waveguide dispersion, D can be written as the sum of two terms,

$D_{\text {intra }}=D_{M}+D_{W}$

where $\mathbf{D}_{\mathbf{M}}$ is the material dispersion and $\mathrm{D}_{\mathbf{W}}$ is the waveguide dispersion.

$$
D_{M}=\frac{-\lambda}{c} \frac{d^{2} n_{e f f}}{d \lambda^{2}}
$$

where $n_{\text {eff }}$ is the effective refractive index given by $[6,7]$

$$
n_{e f f}=\sqrt{f n_{\text {air }} 2+(1-f) n_{\text {silica }} 2}
$$

Where $f$ is the air filling fraction and is equal to (0.9), $\boldsymbol{n}_{\text {air }}$ obtained from the following equation [6]

$$
n_{a i r}=1+.0472326\left(173.3-\frac{1}{\lambda^{2}}\right)^{-1}
$$

But When the holes of the hollow core photonic crystal bandgap fiber filled with materials other than air specially the $n_{\text {air }}$ in equation(9) replaced by $n_{m}$ that obtained by the following Sellmeier formula

$$
n_{m}=1+\frac{c_{2}}{c_{1}-\frac{1}{\lambda^{2}}}+\frac{c_{4}}{c_{3}-\frac{1}{\lambda^{2}}}
$$

Where $\mathbf{c}_{1}, \mathbf{c}_{\mathbf{2}}, \mathbf{c}_{\mathbf{3}}, \mathbf{c}_{4}$ are the Sellmeier coefficients, and $n_{\text {silica }}$ in equation (9) is the refractive index of silica $[8,9$, $10,11,12]$.

\subsubsection{Waveguide Dispersion}

The group velocity of guided optical pulses depends on the wavelength even if material dispersion is negligible. This depend is known as the waveguide dispersion .The contribution of waveguide dispersion
$\mathbf{D}_{\mathbf{W}}$ to the dispersion parameter $\mathbf{D}$ is given by the equation(6). $\mathrm{D}_{\mathrm{W}}$ depends on the index difference $\Delta$ given by the following equation

$$
\Delta=\left(\mathbf{n}_{\mathrm{co}}-\mathbf{n}_{\mathrm{cl}}\right) / \mathbf{n}_{\mathbf{c o}} \ldots \text { (12) }
$$

\subsection{Intermodel Dispersion}

It results from the propagation delay differences between modes within a multimode fiber. As the different modes that constitute a pulse in a multimode fiber travel along the channel at different group velocities, the pulse width at the output is dependent upon the transmission time of the lowest and fastest modes [3].

$$
\begin{aligned}
& \mathbf{D}^{2} \text { total }=\left(\mathbf{D}_{\text {material }}+\mathbf{D}_{\text {waveguide }}\right)^{2} \Delta \\
& \lambda^{2}+\mathrm{D}_{\text {modal }}^{2} \ldots(13)
\end{aligned}
$$

Where $D_{\text {total, }}$ is the intermodal dispersion, $D_{\text {material }}$ is the material dispersion, $D_{\text {waveguide }}$ is the waveguide dispersion, and $D_{\text {modal }}$ is the modal dispersion.

\subsection{Polarization \\ Dispersion (PMD)}

Mode

A fundamental property of an optical signal is its polarization state. Polarization refers to the electric-field orientation of a light signal, which can vary significantly along the length of a fiber. As shown in Figure (1), signal energy at a given wavelength occupies two orthogonal polarization modes. A varying birefringence along its length will cause each polarization mode to travel at a slightly different velocity and the polarization orientation will rotate with distance. The resulting different in propagation modes will result in pulse spreading.

This is the Polarization Mode Dispersion (PMD) . 


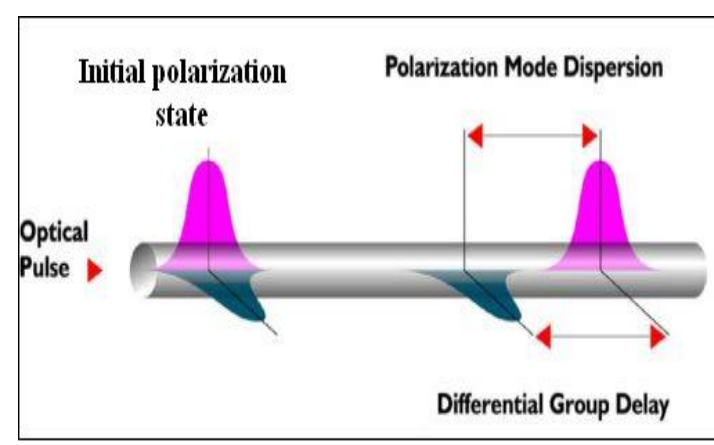

Fig. (1): Variation in polarization states of an optical pulse at it passes through a fiber [8].

\section{Numerical method:}

1-Calculate the effective refractive index $\left(\mathrm{n}_{\text {eff }}\right)$ from equation (9).

2- Plotted the dispersion curve $\left(\mathrm{n}_{\mathrm{eff}}\right.$ with wavelength for PCF when the HCPCFs filled with air).

3- PCF was evacuated and then filled with $\mathrm{N}_{2}$ gas.

4- Calculated the effective refractive index ( $\left.\mathrm{n}_{\text {eff }}\right)$ from equation (9).

5- Plotted the dispersion curve $\left(\mathrm{n}_{\mathrm{eff}}\right.$ with wavelength for PCF when the HCPCFs filled with $\mathrm{N}_{2}$ gas).

6- BGPCFs was evacuated and then filled with He gas.

7- Calculated the effective refractive index $\left(\mathrm{n}_{\text {eff }}\right)$ from equation (9).

8- Plotted the dispersion curve $\left(\mathrm{n}_{\mathrm{eff}}\right.$ with wavelength for PCF when the HCPCFs filled with $\mathrm{He}$ gas).

9- Finally, the material dispersion (D) was calculate of HCPCFs from equation (8), at first when PCF filled with air, second when PCF filled with $\mathrm{N}_{2}$ gas, third when PCF filled with $\mathrm{He}$ gas.

10- Plotted the material dispersion of 19 cell PCF with wavelength in three case above.

\section{Results and Discussion:}

In this work, the material dispersion (D) was calculated of HCPCFs from equation (8), at first we calculate the effective refractive index $\left(\mathrm{n}_{\text {eff }}\right)$ from equation (9) and then plotted the dispersion curve $\left(\mathrm{n}_{\text {eff }}\right.$ with wavelength for photonic crystal fiber when the HCPCFs filled with air), then photonic crystal fiber was evacuated and then filled with $\mathrm{N}_{2}$ gas, and finally BGPCFs was evacuated and then filled with $\mathrm{He}$ gas as shown in figure (2,3 and 4)

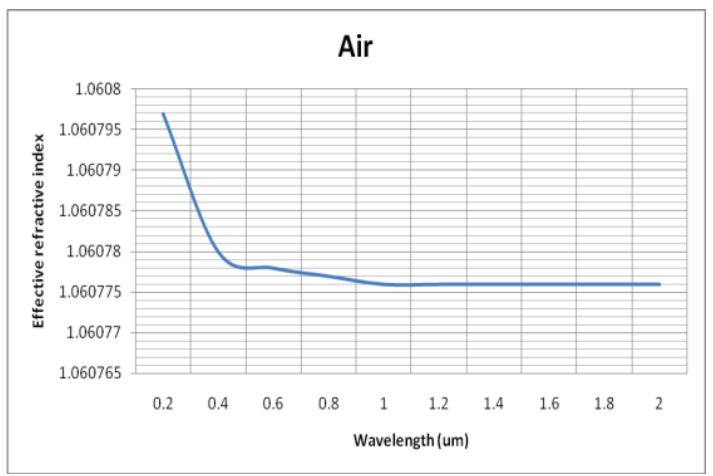

Fig. (2) Effective refractive index of HCPCFs when fiber filled with air as a function of wavelength.

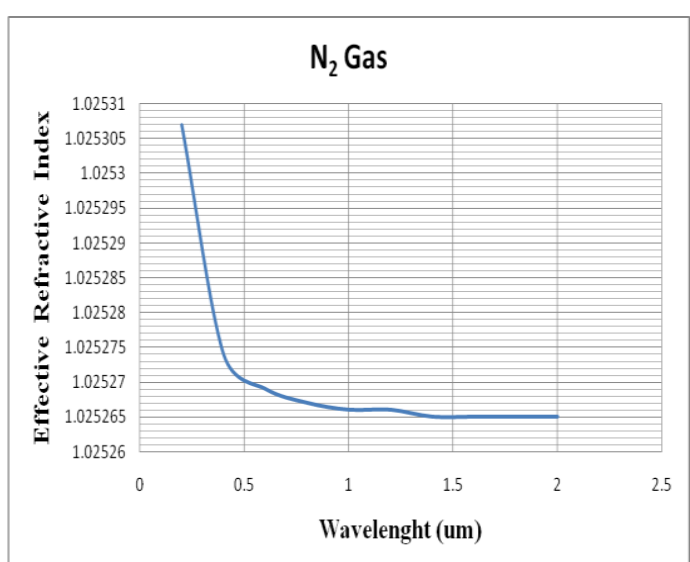

Fig. (3) Effective refractive index of BGPCFs when fiber filled with N2 gas as a function of wavelength.

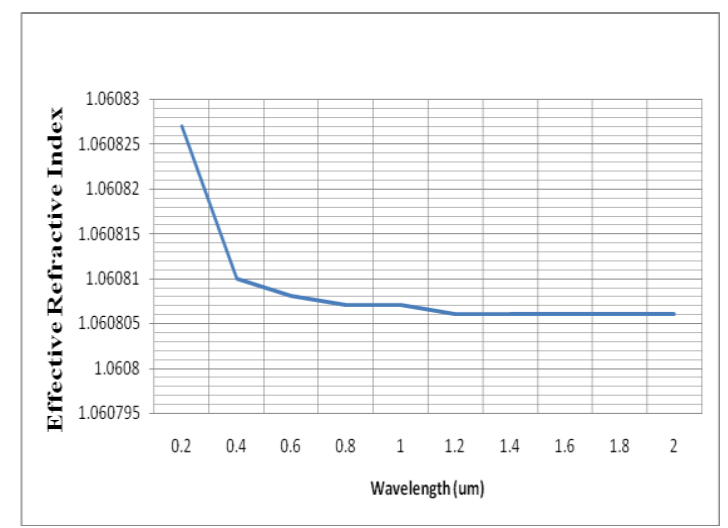

Fig. (4) Effective refractive index of PCF when fiber filled with He gas as a function of wavelength. 
The dispersion properties of the 19 cell BGPCFs were examined in three cases, first when the HCPCFS filled with air as shown in fig. (5)

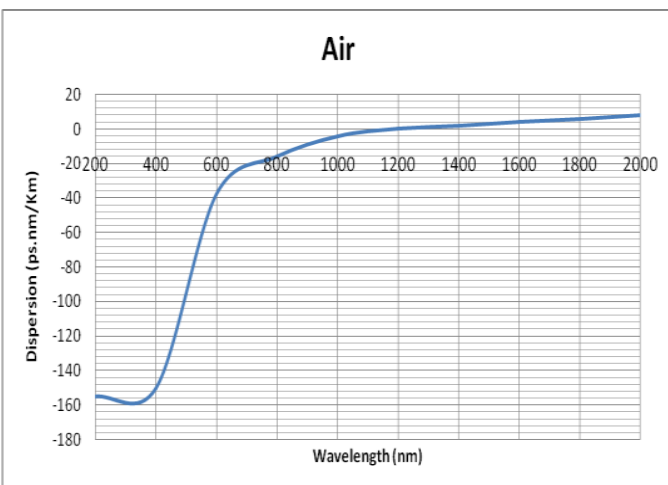

Fig. (5) Material dispersion of 19 cell BGPCFs before fiber filling with gas (i.e filling with air)

From fig.(5) the material dispersion was plotted when the PCF filled with air and it is very clear to notice that the material dispersion is negative for wavelength between (200 to 1000)nm and above this region the material dispersion is positive which is called anomalous dispersion and the waveguide dispersion is dominate in air for negative dispersion.

Second step we calculated the material dispersion from equation (8) when the HCPCFs was evacuated and then filled with $\mathrm{N}_{2}$ gas as shown in fig. (6)

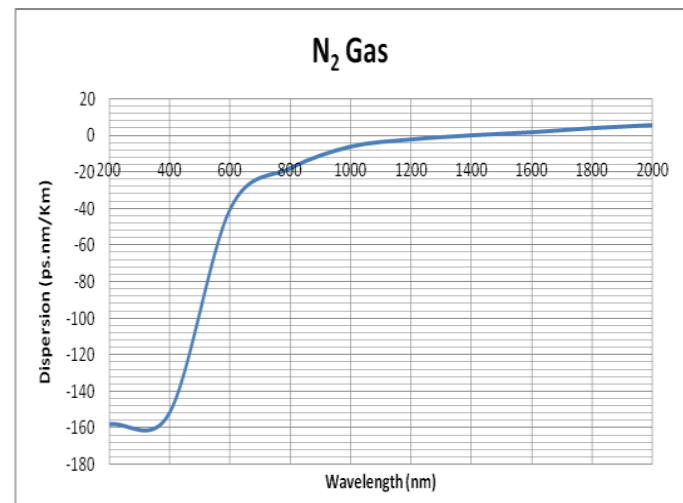

Fig. (6) Material dispersion of 19 cell BGPCFs after fiber filling with N2 gas.

From fig.(6) the material dispersion was plotted when the PCF was evacuated and filled with $\mathrm{N}_{2}$ gas and it is very clear to notice that the material dispersion is negative for wavelength between (200 to 1200)nm and above this region the material dispersion is positive which is called anomalous dispersion.

And finally we calculate the material dispersion from equation (8) when the HCPCFs was evacuated and then filled with He gas as shown in fig. (7)

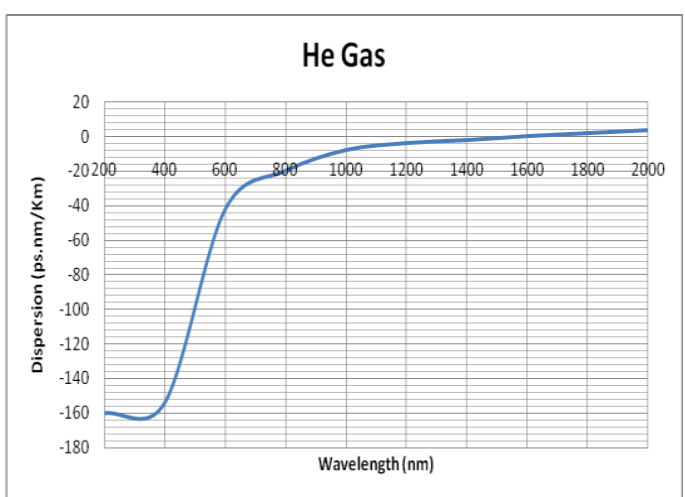

Fig. (7) Material dispersion of 19 cell BGPCFs after filling fiber with $\mathrm{He}$ gas.

From fig.(7) the material dispersion was plotted when the PCF was evacuated and filled with $\mathrm{He}$ gas and it is very clear to notice that the material dispersion is negative for wavelength between (200 to 1400)nm and above this region the material dispersion is positive which is called anomalous dispersion.

\section{Conclusion:}

From above figures it was noticed that the material dispersion is decreased when the holes of PCF filled with $\mathrm{N}_{2}$ gas and $\mathrm{He}$ gas. This will increase the ability to transmit much data when the propagation and dispersion carefully tailorated.

The dispersion properties of photonic crystal fibers (PCFs) were calculated from equation (8), it was observed that the zero dispersion wavelengths for PCF can be engineered to any value by 
altering the size of air holes and separation between them. Nearly zero ultraflattened dispersion from PCFs will promote their application in communication systems, and the high negative chromatic dispersion in PCF will prove useful for dispersion compensating devices.

\section{References:}

1. Sinha R. K. and Shailendra Varshney K. 2003. "Dispersion properties of photonic crystal fibers" ,Microw. Optic. Technol. Lett., 37(2): 155-162.

2. Huttunen A. and TOrma P. 2005. "Effect of wavelength dependence of nonlinearity, gain, and dispersion in photonic crystal fiber amplifiers" ,Optics Express, 13(11): 310-319.

3. Ming-K.Liu M. 1996 "Principle and applications of optical communication",McGraw-Hall:

205-211.

4.Wong K. L. G. 2003 "Non liner optics in photonic crystal fiber", MS.c thesis, University of Auckland.

5. Alkeskjold T. T.; Laegsgaard J.; Bjarklev A.; Hermum D.S.; Anawati A.; Broeng J.; Li J. and Wu S. T. 2005 "Optically Tunable Photonic Bandgap Fiber" Optics Express, 13(6): 2082-2088)
6. Birks T. A.; Bird D. M.; Hedley T. D.; Pottage J. M.; and Russell P. St. J. P. St. J. 2004 "Scaling laws and Vector effects in Bandgap Guiding Fibers", Optics Express .12: 69 -74.

7. Kuhlmey B.T.; Mcphedran R.C.;.desterrke C.M and Robison P.A. 2002 "Microstructured Optical Fibers :where is the edge?",Optics Express, 10(22): 533-541.

8. Okamoto K. 2000 "Fundaments of optical waveguides", academic press: 302-308.

9. Ouyang G., Xu Y., and Yariv A. 2002 "Theoretical study on dispersion compensation in air-core Bragg fibers," Opt. Express 10: 899-908.

10. Engeness T. D., Ibanescu M., Johnson S. G., Weisberg O., Skorobogatiy M., Jacobs S., and Fink Y. 2003 "Dispersion tailoring and compensation by model interactions in OmniGuide fibers," Opt. Express 11:1175-1196.

11. Sinha R. K. and Varshney S. K. 2003 "Dispersion properties of photonic crystal fibers," Microwave and Optical Tech. Lett. 37:129-132.

12. Huttunen A. and T"orm"a P. 2005 "Optimization of dual-core and microstructure fiber geometries for dispersion compensation and large mode area," Opt. Express 13: 627-635. 


\section{التشتت في الليف البلوري الفوتوني المجوف القلب المملوء بالغاز}

حنان جعفر طاهر

معهد الليزر للاراسات العليا

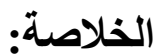

توفر الالياف الفوتونية المجوفة القلب هندسة جديدة لمعرفة وتعزيز الكثير من التأثيرات البصرية اللاخطية. تقام

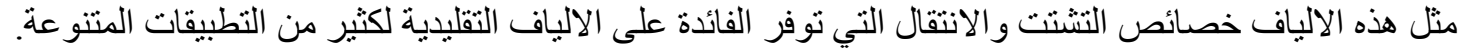

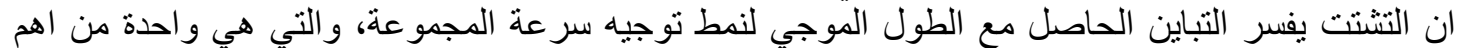

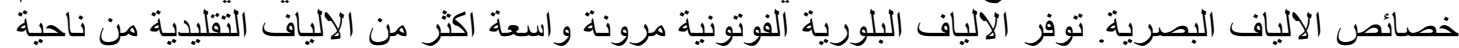

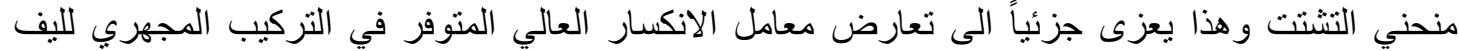

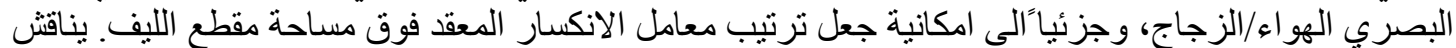

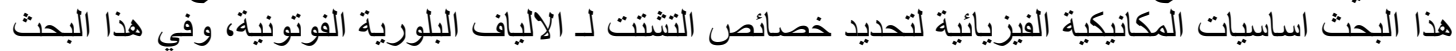

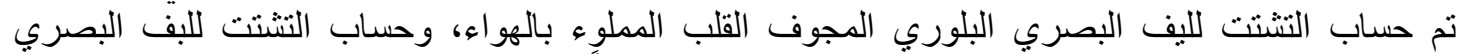

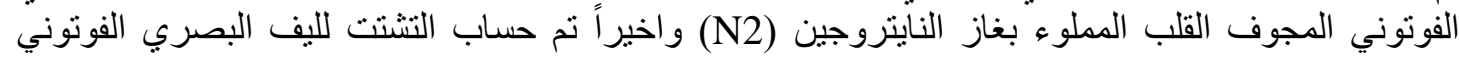
المجوف القلب المملوء بغاز الهليوم (He). 